\title{
REDUCIENDO EL TRAUMA Y LA MORTALIDAD ASOCIADA A LOS ACCIDENTES DE TRÁNSITO EN LOS PEATONES EN EL PERÚ: INTERVENCIONES QUE PUEDEN FUNCIONAR
}

\author{
D. Alex Quistberg ${ }^{1,2, a}$, J. Jaime Miranda ${ }^{3,4, b}$, Beth Ebel $^{1,2,5, c}$
}

\begin{abstract}
RESUMEN
Los peatones peruanos se constituyen como el grupo que sufre la mayor proporción de fatalidades causadas por los accidentes de tránsito en el mundo. El año 2009 los peatones fueron involucrados en $27 \%$ de los accidentes de tránsito en el Perú. Este es un problema significativo para la salud pública del país, pues tiene repercusiones económicas importantes. En este artículo se revisa la evidencia disponible para algunas intervenciones implementadas en otros países y enfocadas a la prevención de los atropellos, las que podrían funcionar bien en Perú. De manera consistente, la implementación de intervenciones tales como el calmar el tráfico en áreas específicas a través de reductores de velocidad ("rompemuelles"), minirotondas ("óvalos"), mejor alumbramiento, calles unidireccionales, desvíos y bloqueos de las calles, tiene una fuerte evidencia en reducir las lesiones y fatalidades en los peatones. Otras intervenciones vinculadas con actividades de educación y hacer cumplir las leyes, podrían tener una función en contextos muy específicos, sin embargo, diseñar y modificar el medio ambiente físico se constituye como una de las intervenciones más importantes.
\end{abstract}

Palabras clave: Accidentes de tránsito; Prevención de accidentes; Legislación; Salud pública; Perú (fuente: DeCS BIREME).

\section{REDUCING PEDESTRIAN DEATHS AND INJURIES DUE TO ROAD TRAFFIC INJURIES IN PERU: INTERVENTIONS THAT CAN WORK}

\begin{abstract}
Pedestrians in Peru are the victims of the greatest proportion of road traffic fatalities in the world. In 2009, pedestrians were involved in $27 \%$ of road traffic incidents in Peru. This is a significant public health problem in Peru and it has important economic effects as well. We review the evidence for some of the solutions and interventions implemented in other countries that may work well in Peru to confront this problem via preventing pedestrian-vehicle incidents. Most importantly, deploying interventions such as area-wide traffic calming through speed bumps, roundabouts, better lighting, one-way streets, detours, and blocking some roads has the strongest evidence for reducing injuries and fatalities in pedestrians. Other interventions related to education and enforcement may have a role as well in very specific contexts, but overall designing and modifying the built environment is the most important intervention.
\end{abstract}

Key words: Accidents, traffic; Accident prevention; Legislation; Public health; Peru (source: MeSH NLM).

\section{INTRODUCCIÓN}

El año 2009, la Organización Mundial de la Salud (OMS) publicó el reporte "Informe sobre la situación mundial de la seguridad vial: es hora de pasar a la acción" (1). A nivel mundial, para el año 2008, los peatones peruanos se constituían como los que sufrían la mayor proporción de fatalidades causadas por los accidentes de tránsito ${ }^{(1)}$. Según las cifras de la Policía Nacional del Perú, los peatones estuvieron involucrados en $27 \%$ de los 86026 accidentes de tránsito registrados en el Perú en el año 2009 (2). Por otra parte, un informe de la Secretaría Técnica del Consejo de Transporte de Lima y Callao publicado en el año 2009 señaló que $32 \%$ de los atropellados en Lima Metropolitana y el Callao fueron menores de 18 años de edad y, 31\% de los atropellados que murieron fueron mayores de 60 años (3).

\footnotetext{
Department of Epidemiology, School of Public Health, University of Washington. Seattle, Washington, EEUU.

Harborview Injury Prevention \& Research Center, University of Washington. Seattle, Washington, EE.UU.

Programa de Investigación en Accidentes de Tránsito, Salud Sin Límites Perú. Lima, Perú.

Facultad de Medicina, Universidad Peruana Cayetano Heredia. Lima, Perú.

Department of Pediatrics, School of Medicine, University of Washington. Seattle, Washington, EEUU

a Magíster en Salud Pública; ${ }^{\text {a }}$ Médico, Magíster y Doctor en Epidemiología; c Médico Pediatra, Magíster en Salud Pública y Magíster en Economía.
}

Recibido: 19-02-10 Aprobado: 28-04-10 
Tabla 1. Matriz de Haddon y las lesiones y fatalidades de los peatones.

\begin{tabular}{|c|c|c|c|c|}
\hline & Peatón & Vehículo & Social & Físico \\
\hline Preacontecimiento & $\begin{array}{l}\text { Demográficos } \\
\text { Intoxicación } \\
\text { Uso de celular } \\
\text { Distracciones } \\
\text { Cruce de calles }\end{array}$ & $\begin{array}{l}\text { Tipo de vehículo } \\
\text { Diseño de vehículo } \\
\text { Capacidad de } \\
\text { frenado } \\
\text { Neumáticos }\end{array}$ & $\begin{array}{l}\text { Respeto al peatón } \\
\text { Cumplimiento de } \\
\text { leyes de tránsito } \\
\text { Normas de } \\
\text { comportamiento }\end{array}$ & $\begin{array}{l}\text { Alumbrado público } \\
\text { Densidad de tráfico } \\
\text { Densidad peatonal } \\
\text { Tiempo en el día } \\
\text { Señalización } \\
\text { Disminución de tráfico } \\
\text { Pavimento } \\
\text { Ambiente construido seguro } \\
\text { Mal diseño de vías }\end{array}$ \\
\hline Acontecimiento & $\begin{array}{l}\text { Negligencia } \\
\text { Imprudencia } \\
\text { Distracción } \\
\text { Capacidad reactiva } \\
\text { Huir luego de chocar }\end{array}$ & $\begin{array}{l}\text { Momento } \\
\text { Fuerza de impacto } \\
\text { Parte que golpea }\end{array}$ & $\begin{array}{l}\text { Conocimientos de } \\
\text { primeros auxilios }\end{array}$ & $\begin{array}{l}\text { Hospital más cercano } \\
\text { Servicio médico de emergencia } \\
\text { Facilidades de cuidados de } \\
\text { emergencias }\end{array}$ \\
\hline Postacontecimiento & $\begin{array}{l}\text { Capacidad de } \\
\text { recuperación } \\
\text { Recuperación }\end{array}$ & $\begin{array}{l}\text { Gravedad del } \\
\text { impacto }\end{array}$ & $\begin{array}{l}\text { Aseguramiento } \\
\text { en salud } \\
\text { Rehabilitación }\end{array}$ & Rehabilitación \\
\hline
\end{tabular}

Adaptado de CW Runyan ${ }^{(4,5)}$.

Este es un problema que requiere la atención, apoyo y trabajo de aquellos responsables e involucrados con la salud pública, de los prestadores de servicios de salud y cuidado médico, de los ingenieros y diseñadores de las vías de transporte, así como de los representantes del gobierno. A la fecha, se han puesto en práctica muchas soluciones que, potencialmente, podrían implementarse y desarrollarse en el Perú para mejorar la seguridad vial de los peatones. Tomando como base la experiencia procedente de países desarrollados, este artículo tiene por objetivo revisar la evidencia vinculada con las intervenciones orientadas a la prevención de atropellos - con un énfasis en su impacto en los peatones - y sus potenciales aplicaciones en el Perú.

Conocer y entender las causas de los atropellos en el Perú es fundamental para aproximarse al problema. En las décadas de los años 60 y 70 el Dr. William Haddon, el primer directordelaAdministración Nacional deSeguridad de Transportes de Carreteras de los Estados Unidos (National Highway Transportation Safety Administration), describió un esquema -muy utilizado desde entoncespara desarrollar medidas preventivas o para tratar a las lesiones provocadas por los accidentes de tránsito; mundialmente conocida como la matriz de Haddon (Tabla 1); se compone de tres filas (preacontecimiento, acontecimiento y postacontecimiento) y de cuatro columnas (individuo, vector, medioambiente social, y medioambiente físico) ${ }^{(4,5)}$. El modelo típico para entender las lesiones provocadas por un accidente de tránsito, permitiendo conceptualizar la interrelación entre estos elementos, se muestra en la Figura $1^{(6)}$. Analizando la matriz de Hadddon se desprende que, para establecer acciones en términos de prevención de lesiones vinculadas a los accidentes de tránsito resulta necesario enfocarse principalmente en modificar los factores de la primera fila (preacontecimiento) y también algunos de la segunda fila (eventos). Según la experiencia de los Estados Unidos, para la prevención de las lesiones por accidentes de tránsito existen tres enfoques tradicionales, comúnmente referidos como las tres E: educación, hacer cumplir las leyes e ingeniería (Education, Enforcement y Engineering).

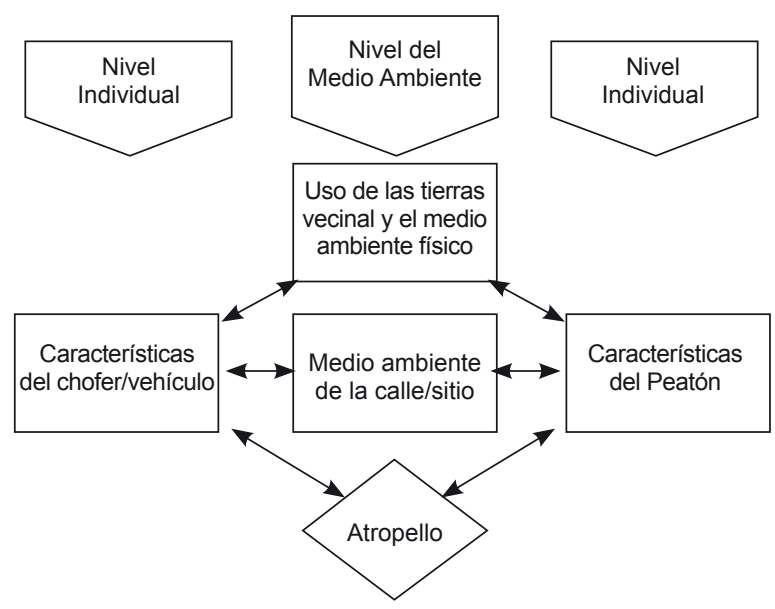

Figura 1. Modelo de factores relacionados con la seguridad vial de los peatones ${ }^{(6)}$. 


\section{MODIFICACIÓNDEATRIBUTOS INDIVIDUALES: CENTRÁNDOSE EN EL PEATÓN}

En la experiencia de los Estados Unidos, la mayoría de estos cambios fueron impulsados a través de estrategias educacionales; entre ellas, se desarrollaron campañas sociales mediante anuncios televisivos o por afiches en las comunidades, programas de capacitación en las escuelas yeducación de los padres acerca de la seguridad vial. Una revisión Cochrane sobre la efectividad estos tipos de programas, que analizó ensayos controlados con asignaciones al azar, concluyó que con una educación peatonal se pueden mejorar tanto el conocimiento de la seguridad vial como el comportamiento al cruzar las calles en los niños, pero la evidencia no es muy fuerte por la falta de estudios aleatorizados de alta calidad ${ }^{(7)}$. Entre los adultos no se demostró un efecto de cambio en actitudes o comportamientos, ni tampoco un cambio en la incidencia de las lesiones. Estos resultados, muy pobres en términos de magnitud de impacto y relevancia para salud pública, no son concluyentes dado que los estudios incluidos fueron pocos y no siempre fueron muy rigurosos; adicionalmente, ninguno fue realizado en países de ingresos bajos o medianos.

¿De qué manera es relevante esta evidencia para nuestro medio y nuestra región? En el Perú, los niños representan alrededor de $30 \%$ de los atropellados. Por tanto, podría resultar intuitivo que los programas de educación peatonal o programas orientados al público en general pudiera ser un camino para disminuir el volumen de lesiones por accidentes de tránsito. Dada la evidencia limitada, se necesita estudios locales antes de embarcarse en un programa de gran envergadura. Es importante recordar que los niños pueden aprender el comportamiento correcto, pero quizás no necesariamente siempre hacen lo correcto.

La Academia Americana de Pediatría ha recomendado que, por lo menos, los médicos pediatras sean los que aconsejen a los padres de los peligros viales en los niños. Esta recomendación se basa en el hecho de que los padres muchas veces no comprenden que los niños pequeños todavía no han desarrollado la habilidad de alerta ante la presencia o aparición de vehículos cuando juegan o cruzan las calles ${ }^{(8)}$. En el Perú se han puesto en práctica algunos programas de educación de seguridad vial (Programa Educativo de Seguridad Vial ${ }^{(9)}$ y Transitando). Dado que las estadísticas reportan que $53 \%$ de los choques son atribuibles - un tema aparte es la calidad de la recolección de esta información-a imprudencias de los peatones en Lima y Callao ${ }^{(3)}$, suena lógico que este tipo de iniciativas podrían beneficiar al Perú. Sin embargo, hay que mantener el escepticismo sobre su impacto real pues los diseños aleatorizados han demostrado un nulo impacto, y la evaluación de efectividad de los programas de educación en nuestro medio se hacen imperativos.

\section{MODIFICACIÓN DE ATRIBUTOS DEL VECTOR: CENTRÁNDOSE EN LOS VEHÍCULOS}

En términos del vector, es evidente que la velocidad es el factor más importante que debe cambiarse en el vehículo. Idealmente, en zonas residenciales la velocidad no debe exceder los $35 \mathrm{~km} / \mathrm{h}^{(8,10)}$. Aun en otras zonas donde hay peatones, las velocidades no deben exceder los $50 \mathrm{~km} / \mathrm{h}$ porque la probabilidad de muerte de un peatón atropellado a esta velocidad es de $40 \%$, mientras que a los $65 \mathrm{~km} / \mathrm{h}$ es más del $80 \%$. Un aspecto por considerar es que los choferes suelen conducir un poco más rápido de lo permitido tanto en vías rápidas como en zonas residenciales ${ }^{(11,12)}$.

Un trabajo realizado en Londres, ${ }^{(10)}$ demostró que la introducción de límites de velocidad a $32 \mathrm{~km} / \mathrm{h}$ en zonas urbanas redujo el número de víctimas en $42 \%$ (IC 95\%: 36-48\%). Este impacto fue acompañado con una reducción del número de víctimas en las zonas aledañas de $8 \%(4-12 \%)$. Esta reducción de velocidad no sólo tiene un impacto positivo en los peatones al reducir su exposición al riesgo, sino que también reduce el riesgo de presentar daño tanto en choferes como en pasajeros. El poder implementar zonas y límites de velocidad similares a lo descrito anteriormente requiere poner en funcionamiento medidas que involucran intervenciones vinculadas con las áreas legales y de ingeniería, las cuales serán abordadas más adelante. Hay varias medidas de bajo costo que han tenido éxito y que podrían ser usadas en países como Perú, incluyendo el reducir la velocidad a través de reductores de velocidad ("rompemuelles") o variaciones en estos ${ }^{(13)}$.

Otra característica importante relacionada con el vector es el tipo de vehículo involucrado en el accidente de tránsito, sea auto, camioneta o bus. En general, mientras más grande y alto sea el vehículo, más grave es el choque, por existir una mayor probabilidad de lesión en la cabeza ${ }^{(8,14,15)}$. También la altura del parachoques puede determinar el tipo de lesión que sufra el peatón (16). Modificar estos factores resulta más difícil, ya que dependen de los fabricantes; sin embargo, esto ya es un requisito para los autos particulares que se venden en Europa ${ }^{(8)}$.

Resulta positivo el saber que en aspectos de ingeniería automovilística ya existen algunos modelos de autos, sobre todo aquellos destinados para un uso particular, que son diseñados teniendo en cuenta el reducir 
las lesiones de los peatones en caso de atropello. Estas modificaciones al vehículo particular incluyen la detección de peatones por LIDAR (Light Detection and Ranging o Laser Imaging Detection and Ranking, una tecnología que permite determinar la distancia desde un emisor láser a un objeto o superficie, utilizando un haz láser pulsado) que induce el vehículo a frenar automáticamente ${ }^{(17,18)}$, disminuyendo la altura de los parachoques (19), acondicionando bolsas de aire adicionales en los parabrisas en los vehículos (20), haciendo que los parachoques sean más amoldables (19), e instalando sensores para ayudar a los choferes a detectar con más facilidad a los peatones ${ }^{(19)}$

Los autos particulares son responsables del 34,4\% del total de muertes peatonales en el área de Lima y Callao; $36,0 \%$ son causados por vehículos de transporte público y $19,9 \%$ por camiones y camionetas ${ }^{(3)}$. Vale la pena notar que, según el vector o vehículo, los de transporte público pueden involucrar a autos particulares que funcionen como colectivos o taxis. En tal sentido, además de las mejoras señaladas anteriormente, que están destinadas a los vehículos particulares, resultan necesarias otras modificaciones importantes a los otros medios de transporte para mejorar la prevención de lesiones en los peatones. La mayoría de las investigaciones disponibles en esta materia se enfocan en la mejoría de la seguridad de los autos particulares porque en los países de altos ingresos estos se constituyen como el vehículo más común, no son confundidos con trasporte particular y, por ende, en las estadísticas son reportados completamente aparte. Además, queda pendiente, en nuestro medio, investigar cómo modificar o hacer más seguros a los vehículos de transporte público, como omnibuses, motocarros ("mototaxis") y camionetas rurales, cuyas respuestas locales pueden resultar relevantes no sólo para el Perú sino también para otros países con contextos similares.

\section{MODIFICACIÓN DE ATRIBUTOS DEL MEDIOAMBIENTE SOCIAL}

Se puede pensar que las estrategias educativas dirigidas a poner énfasis en el hacer cumplir las leyes, son las más importantes cuando se trata de modificar factores vinculados con el medioambiente social; pero como ya se mencionó, no existe suficiente evidencia de efectividad en aquellas intervenciones orientadas a cambiar los comportamientos personales por medio de campañas educativas ${ }^{(7)}$. En cambio, la evidencia no es tan clara con relación al impacto de intervenciones educativas a nivel comunitario. En un ensayo se demostró que los comportamientos de los peatones al usar cruces peatonales, y de los choferes de ceder el paso a los peatones, mejoraron -en generalinmediatamente con una campaña enfocada a una comunidad específica, pero las intervenciones tienen que ser mantenidas o repetidas a través del tiempo basados en las observaciones a un año después ${ }^{(21)}$. La campaña incluyó acciones comunitarias orientadas a mejorar el nivel de conocimiento de choferes y peatones con respecto a la seguridad vial, folletos de información, demostraciones de las pruebas de choques con muñecos, contratos de compromiso y cobertura en medios de comunicación, tanto en periódicos como en canales de televisión. Una limitante de este estudio es que la comunidad intervenida fue un pueblo universitario, en donde probablemente la comunidad podía haber tenido una predisposición al cambio mucho mayor durante la ejecución y postintervención. Otra limitante importante es que las variables resultados no evaluaron el impacto de la intervención en el número de atropellos.

Para mayor efectividad, se propone que las acciones relacionadas con ejercer el cumplimiento de las leyes deben acompañase por las intervenciones educativas. Con respeto al tema de rigurosidad en la aplicación de las leyes, hay muy pocos ensayos y evidencia para apoyar el uso de ésta como intervención única para prevenir lesiones peatonales, pero la mayoría de los expertos en seguridad vial concuerdan en que los policías son vitales para aminorar el riesgo peatonal ${ }^{(22)}$. Un estudio en Irán encontró que las campañas de educación acompañadas con el hacer cumplir la ley por parte de la policía, redujo la tasa de muerte causada por accidentes de tránsito, de 38,2 por 100000 habitantes en 2004 a 31,8 en $2007^{(23)}$. Es posible que existan otras explicaciones para el efecto observado, pero la aplicación de leyes para la protección de los peatones sugiere tener un impacto positivo.

Existen leyes específicas que protegen a los peatones, las cuales deben respetarse, por ejemplo los peatones solamente deben cruzar por los lugares designados y los conductores deben ceder el paso al peatón. El lograr que estas leyes se respeten requiere no solamente educar en seguridad vial sino la puesta en práctica de medidas de sanción más drásticas por su incumplimiento. En países desarrollados funcionan las sanciones, tal como multar por una falta o establecer trabajos comunitarios como reparación por el daño ocasionado. Su efectividad en otros contextos, en donde el sistema legal de sanciones tiene serias limitaciones como el nuestro, queda por definirse.

Finalmente, hay otro problema social creciente para los choferes y los peatones, que es el uso de celulares al conducir o cruzar la calle. Estudios en la última década demuestran que los conductores que usan el celular mientras manejan tienen el mismo riesgo de accidentarse 
que quienes tienen una concentración de alcohol en la sangre de 0,08 o más alto ${ }^{(24-26)}$. Recientemente se han explorado los efectos de conversar por celular en los peatones mientras caminan y se ha encontrado que aquellos peatones que caminan conversando dan pasos inseguros ${ }^{(27,28)}$ y se demoran más en cruzar que los que no están conversando ${ }^{(29)}$, inclusive los que van escuchando música portando dispositivos de música portátiles ${ }^{(29)}$. En los Estados Unidos y, recientemente en el Perú, muchos estados y ciudades han restringido el uso de celulares al conducir (conversar y mandar mensajes de texto), pero la efectividad de estas leyes necesita de evaluación. Cómo enfrentar este problema potencial en los peatones, requiere de soluciones innovadoras.

\section{MODIFICACIÓN DE ATRIBUTOS DEL MEDIOAMBIENTE FÍSICO}

La evidencia más sólida disponible para la prevención de las lesiones por accidentes de tránsito en los peatones está vinculada con la modificación de los atributos físicos. Además, por cierto, es necesario rescatar que pueden constituir las intervenciones más caras de implementarse. A largo plazo, en contraste, estos programas pueden resultar menos costosos que otras intervenciones que han sido mencionadas. Aunque inicialmente los costos de algunas de estas intervenciones resultan altos y requieren un buen planeamiento, los efectos positivos y saludables adicionales no son sólo observables en la reducción de las lesiones sino también podrían tener un impacto en reducir la obesidad y mejorar el medio ambiente. El mejor momento para implementarlas es durante las fases de planificación y diseño de obras de infraestructura, pero, los lugares ya establecidos pueden y deberían ser modificados para asegurar un ambiente seguro para el peatón. Algunas veces, para mejorar la seguridad vial de los peatones $-\mathrm{y}$ la de los choferes también- se puede modificar una o dos cosas en el diseño de una zona. Sin embargo, la mayoría de veces las modificaciones necesarias requieren cambiar una variedad de características de las calles o el entorno físico, lo cual encarece su implementación.

Como explicamos anteriormente, la velocidad del vehículoes uno de los factores de riesgo más importantes para la fatalidad de un peatón. El calmar el tráfico en áreas específicas es una intervención que promete ser preventiva de las lesiones y fatalidades de los peatones porque su función es reducir la velocidad y el volumen de tráfico en las zonas urbanas y residenciales (30). Esta intervención puede incluir reductores de velocidad, minirotondas, mejor alumbramiento, calles unidireccionales, desvíos y bloqueos de las calles.
A pesar de que resulta aparentemente beneficioso no existe evidencia, especialmente procedente de países de ingresos bajos o medianos, que respalden la efectividad de estas propuestas. Aunque su impacto no ha sido demostrado en términos de mortalidad o reducción de lesiones por accidentes de tránsito, por lo menos, varios estudios han demostrado que estas estrategias de implementación en las vías funcionan bien en reducir la velocidad de circulación del vehículo en comparación a las zonas sin estas intervenciones $(22,31)$. Otra manera de disminuir la velocidad del tráfico es hacer que los carriles sean más angostos y colocar árboles al lado de las calles ${ }^{(32)}$. El razonamiento detrás de estas medidas, que parecerían que pudieran facilitar la producción de choques, es que sirven para hacer que los choferes sean más prudentes y puedan manejar más despacio.

Por parte de los peatones, la exposición al riesgo de sufrir un accidente de tránsito se explica por el hecho de que todos los peatones tienen que, en algún momento, cruzar una calle para llegar a su destino. Existen varias maneras de proteger a los peatones de los vehículos mientras cruzan la calle, las más eficaces separan la circulación de los peatones de la circulación de los vehículos por tiempo, espacio, o ambos ${ }^{(22,31)}$. Ejemplo de ello es la señalización para los peatones, puentes peatonales, barreras en las rayas divisorias, veredas, e islas de seguridad ${ }^{(31)}$. Perú ya ha implementado algunas de estas estrategias, pero no en todas las zonas donde se necesitan ${ }^{(3)}$. A pesar de la implementación realizada, no existen evaluaciones de por medio que den señales de su efectividad, lo cual limita la generación de evidencia para su expansión a otras zonas. Un estudio del reciente Programa de Investigación en Accidentes de Tránsito, ejecutado por Salud Sin Límites Perú, por encargo del Instituto Nacional de Salud, encontró que la presencia de puentes peatonales per se no es lo primordial sino cuál es el beneficio que estos traen para los peatones. Ciertamente, el beneficio parece ser limitado y, por ende, los peatones no hacen un uso completo de este tipo de infraestructura ${ }^{(33,34)}$.

Muchas veces, a pesar de la existencia de algunas de estas intervenciones, no es suficiente para proteger la circulación peatonal. La Administración Federal de Carreteras (Federal Highway Administration) de los Estados Unidos, ha elaborado una página Web (http:// www.walkinginfo.org/pedsafe/) donde muestra la utilización de estas intervenciones y cómo se podrían seleccionar las más adecuadas en varias situaciones ${ }^{(35)}$. Otras medidas que acompañen a las mencionadas anteriormente son las que hacen a los peatones más visibles para los choferes (31); estos incluyen cruces peatonales pintados, con iluminación aumentada y 
con el traslado de paraderos peatonales lejos de los cruces.

En Perú, se pueden encontrar varios tipos de estas intervenciones, pero no siempre están implementadas juntas a fin de aumentar el efecto máximo, o no están ubicadas en los sitios donde son más necesarias. No conocemos la efectividad en estas intervenciones en reducir los atropellos porque hay una carencia de investigaciones al respecto. Estudiar el medio ambiente físico puede resultar difícil puesto que se necesita controlar una serie de factores temporales tal como el flujo del tráfico vehicular y peatonal, pero existen varios ejemplos de trabajos exitosos ${ }^{(36-38)}$. Conocer y entender la efectividad de estos tipos de intervenciones podría ser muy importante para disminuir el problema peatonal. Por lo menos un estudio sobre los niños peatones mostró que la velocidad de los vehículos, la densidad del tráfico vehicular, y los carriles no bien señalizados son riesgos para la ocurrencia de atropellos en niños peruanos ${ }^{(39)}$.

\section{A MANERA DE CONCLUSIÓN}

En conclusión, la seguridad vial es un tema crucial para el Perú y debe dedicársele una alta importancia. De lo contrario, miles de peatones morirán cada año y decenas de miles más sufrirán lesiones y el problema aumentará mientras el parque automotor siga creciendo. Es muy difícil ser un peatón en las zonas urbanas del Perú.

En este artículo se han presentado varias soluciones que pueden implementarse o complementar a lo ya existente y se ha discutido la evidencia disponible para algunas de ellas. Algunas son más prácticas y fáciles de utilizar, pero las más importantes son las que modifican al medio ambiente físico. Muchas veces en los cruces de las calles y avenidas no existen indicaciones para cuando un peatón puede cruzar, los choferes no tienen respeto hacia los peatones, y el acceso a muchos sitios es peligroso porque no hay opciones seguras.

En las calles y avenidas hacen falta medidas para mejorar la seguridad de los peatones y sobre todo, separarlos del flujo vehicular. En los pueblos jóvenes y zonas residenciales puede resultar importante implementar aquellas intervenciones orientadas a calmar el tráfico cerca de las calles y avenidas. Muchos reductores de velocidad o "rompemuelles" han sido instalados en calles adyacentes a lugares de tráfico alto, sin embargo al revisar las estadísticas resulta evidente que es necesario hacer más para proteger a los peatones.

El Perú puede liderar en contribuir al conocimiento sobre la efectividad de estas intervenciones como un país de ingreso bajo y mediano. Existen muy pocos estudios correctamente realizados sobre este tema y es posible que estas intervenciones tengan un efecto diferente en contextos similares al Perú, según la evidencia de los trabajos encontrados en la literatura ${ }^{(40,41)}$. Sin embargo, antes de su implementación, es necesario asegurar que las intervenciones que se proponen sean apropiadas para las coyunturas de un país como Perú, y que sean medidas de manera rigurosa para asegurarse que realmente producen los resultados y beneficios esperados.

\section{AGRADECIMIENTOS}

Nuestro agradecimiento a Julio Antonio Poterico Rojas por su apoyo editorial en la elaboración de este manuscrito.

\section{Fuente de Financiamiento}

JJM fue el investigador principal del Programa de Investigación en Accidentes de Tránsito (PIAT) que fue financiado por el Instituto Nacional Salud de Perú y ejecutado por la ONG Salud Sin Límites Perú.

\section{Conflictos de Interés}

Los autores declaran no tener conflictos de interés en la publicación de este artículo.

\section{REFERENCIAS BIBLIOGRÁFICAS}

1. Organización Mundial de la Salud. Informe sobre la situación de la seguridad vial: Es hora de pasar a la acción. Geneva: Organización Mundial de la Salud; 2009.

2. Policía Nacional de Perú. Accidentes de tránsito 2009. Lima: Ministerio de Defensa de Perú; 2010.

3. Secretaría Técnica del Consejo de Transporte de Lima y Callao. La vulnerabilidad de los peatones en la vialidad del área metropolitana de Lima y Callao. Lima: Ministerio de Transportes y Comunicaciones; 2009

4. Runyan CW. Using the Haddon matrix: introducing the third dimension. Injury Prevention. 1998; 4(4): 302-7.

5. Runyan CW. Introduction: Back to the future - revisiting Haddon's conceptualization of injury epidemiology and prevention. Epidemiol Rev. 2003; 25: 60-64.

6. Moudon AV, Lin L, Hurvitz P. Managing pedestrian safety I: injury severity. Seattle, WA: Washington State Transportation Center (TRAC), University of Washington; 2007.

7. Duperrex O, Bunn F, Roberts I. Safety education of pedestrians for injury prevention: a systematic review of randomized controlled trials. BMJ. 2002; 324(7346): 1129-31.

8. Committee on Injury, Violence, and Poison Prevention, American Academy of Pediatrics. Policy statement -pedestrian safety. Pediatrics. 2009; 124(2): 802-12.

9. Perú, Consejo Nacional de Seguridad Vial. Guía de educación en seguridad vial para profesores y tutores de primaria. Lima: MINEDU, MTC; 2008. 
10. Grundy C, Steinbach R, Edwards $P$, Green J, Armstrong B, Wilkinson P. Effect of $20 \mathrm{mph}$ traffic speed zones on road injuries in London, 1986-2006: controlled interrupted time series analysis. BMJ. 2009; 339: b4469.

11. Damsere-Derry J, Afukaar FK, Donkor P, Mock C. Study of vehicle speeds on a major highway in Ghana: Implication for monitoring and control. Traffic Inj Prev. 2007; 8(2): 142-46.

12. Damsere-Derry J, Afukaar FK, Donkor P, Mock C. Assessment of vehicle speeds on different categories of roadways in Ghana. Int J Inj Contr Saf Promot. 2008; 15(2): 82-91.

13. Afukaar FK. Speed control in developing countries: issues challenges and opportunities in reducing road traffic injuries. Int J Inj Contr Saf Promot. 2003; 10(1-2): 77-81.

14. Roudsari BS, Mock C, Kaufman R, Grossman D, Henary BY, Crandall J. Pedestrian crashes: higher injury severity and mortality rate for light truck vehicles compared with passenger vehicles. Inj Prev. 2004; 10(3): 154-58.

15. Paulozzi LJ. United States pedestrian fatality rates by vehicle type. Inj Prev. 2005; 11(4): 232-36.

16. Matsui Y. Effects of vehicle bumper height and impact velocity on type of lower extremity injury in vehicle-pedestrian accidents. Accid Anal Prev. 2005; 37(4): 633-40.

17. Broggi A, Cerri P, Ghidoni S, Grisleri P, Jung HG. A new approach to urban pedestrian detection for automatic braking. IEEE Trans Intell Transp Syst. 2009; 10(4): 594605.

18. Premebida C, Ludwig O, Nunes U. LIDAR and visionbased pedestrian detection system. J Field Robot. 2009; 26(9): 696-711.

19. Gandhi T, Trivedi MM. Pedestrian protection systems: issues, survey, and challenges. IEEE Trans Intell Transp Syst. 2007; 8(3): 413-430.

20. Untaroiu CD, Shin J, Crandall JR. A design optimization approach of vehicle hood for pedestrian protection. Int J Crashworthiness. 2007; 12(6): 581-89.

21. Boyce TE, Geller ES. A community-wide intervention to improve pedestrian safety - Guidelines for institutionalizing large-scale behavior change. Environ Behav. 2000; 32(4): 502-20.

22. Campbell BJ, Zegeer CV, Huang HH, Cynecki MJ. A review of pedestrian safety research in the United States and abroad. Mclean, VA: Federal Highway Administration; 2004.

23. Soori H, Royanian M, Zali AR, Movahedinejad A. Road traffic injuries in Iran: the role of interventions implemented by traffic police. Traffic Inj Prev. 2009; 10(4): 375-78.

24. Strayer DL, Drews FA, Crouch DJ. A comparison of the cell phone driver and the drunk driver. Hum Factors. 2006; 48(2): 381-91.

25. Drews FA, Yazdani H, Godfrey CN, Cooper JM, Strayer DL. Text messaging during simulated driving. Hum Factors. 2009; 51(5): 762-70.

26. Loeb PD, Clarke WA, Anderson R. The impact of cell phones on motor vehicle fatalities. Appl Econ. 2009; 41(22): 2905-14.

27. Hatfield J, Murphy S. The effects of mobile phone use on pedestrian crossing behaviour at signalised and unsignalised intersections. Accid Anal Prev. 2007; 39(1): 197-205.
28. Nasar J, Hecht P, Wener R. Mobile telephones, distracted. attention, and pedestrian safety. Accid Anal Prev. 2008; 40(1): 69-75.

29. Neider MB, McCarley JS, Crowell JA, Kaczmarski H, Kramer AF. Pedestrians, vehicles, and cell phones. Accid Anal Prev. 2010;42:589-594.

30. Bunn F, Collier T, Frost C, Ker K, Roberts I, Wentz R. Areawide traffic calming for preventing traffic related injuries. Cochrane Database Syst Rev. 2003; (1): CD003110.

31. Retting RA, Ferguson SA, McCartt AT. A review of evidence-based traffic engineering measures designed to reduce pedestrian-motor vehicle crashes. Am J Public Health. 2003; 93(9): 1456-63.

32. Ewing R, Dumbaugh $\mathbf{E}$. The built environment and traffic safety: a review of empirical evidence. J Plann Lit. 2009; 23(4): 347-67.

33. Huicho L, Miranda JJ, Luna D, Paca A, López L, Rosales E, et al. Estudio CAP, nivel de sensibilización, movilización, participación y fortalecimiento de organizaciones comunitarias en la prevención de daños y riesgos relacionados a accidentes de tránsito / Estudio Cualitativo, Grupos focales [Informe Técnico]. Lima: Instituto Nacional de Salud, Salud Sin Límites Perú; 2009.

34. Huicho L, Miranda JJ, Luna D, Paca A, López L, Rosales E, et al. Estudio CAP, nivel de sensibilización, movilización, participación y fortalecimiento de organizaciones comunitarias en la prevención de daños y riesgos relacionados a accidentes de tránsito / Encuesta en padres e hijos [Informe Técnico]. Lima: Instituto Nacional de Salud, Salud sin Límites Perú; 2009.

35. Pedestrian and Bicycle Information Center. PEDSAFE: Pedestrian safety guide and countermeasure selection system. Washington DC: Federal Highway Administration; 2004.

36. Koepsell T, McCloskey L, Wolf M, Moudon AV, Buchner D, Kraus J, et al. Crosswalk markings and the risk of pedestrian-motor vehicle collisions in older pedestrians. JAMA. 2002; 288(17): 2136-43.

37. Sebert Kuhlmann AK, Brett J, Thomas D, R. Sain SR. Environmental characteristics associated with pedestrianmotor vehicle collisions in Denver, Colorado. Am J Public Health. 2009; 99(9): 1632-37.

38. Harruff RC, Avery A, Alter-Pandya AS. Analysis of circumstances and injuries in 217 pedestrian traffic fatalities. Accid Anal Prev. 1998; 30(1): 11-20.

39. Donroe J, Tincopa M, Gilman RH, Brugge D, Moore DAJ. Pedestrian road traffic injuries in urban Peruvian children and adolescents: case control analyses of personal and environmental risk factors. PLoS One. 2008; 3(9): e3166.

40. De Andrade SM, Soares DA, Matsuo T, Liberatti CLB, Iwakura MLH. Road injury-related mortality in a mediumsized Brazilian city after some preventive interventions. Traffic Inj Prev. 2008; 9(5): 450-55.

41. Híjar M, Trostle J, Bronfman M. Pedestrian injuries in Mexico: a multi-method approach. Soc Sci Med. 2003; 7(11): 2149-59.

Correspondencia: D. Alex Quistberg

Correo electrónico: aquistbe@uw.edu 